\title{
Detection of Small Airway Dysfunction in Asymptomatic Smokers with Preserved Spirometry: The Value of the Impulse Oscillometry System
}

\author{
Roberta Pisi (iD) \\ Marina Aiello (D) ${ }^{1}$ \\ Annalisa Frizzelli' \\ Luigino Calzetta (1D) \\ Laura Marchi' \\ Giuseppina Bertorelli' \\ Giovanna Pisi ${ }^{2}$ \\ Alfredo Chetta (1) 1 \\ 'Department of Medicine and Surgery, \\ Respiratory Disease and Lung Function \\ Unit, University of Parma, Parma, Italy; \\ ${ }^{2}$ Department of Pediatrics, Cystic \\ Fibrosis Center, University Hospital, \\ Parma, Italy
}

\begin{abstract}
Purpose: Smoking-induced bronchiolitis with progressive small airway dysfunction (SAD) is a leading cause of chronic obstructive pulmonary disease. We investigated the value of using the impulse oscillometry system (IOS) to detect SAD in asymptomatic smokers with preserved spirometry.
\end{abstract}

Patients and Methods: We included 75 asymptomatic smokers ( 37 females, mean age 47 \pm 12 years, $26 \pm 17$ pack/year) with preserved spirometry [forced expiratory volume at 1st second (FEV1)/forced vital capacity (FVC) $\geq 0.70$ and normal FVC] and 34 neversmokers (19 females, mean age $42 \pm 15$ years).

Results: In smokers, pack/years were significantly related to spirometry and IOS parameters $(p<0.05)$. The values of the fall in resistance from $5 \mathrm{~Hz}$ to $20 \mathrm{~Hz}$ (R5 - R20) were significantly and inversely related to the values of the ratio of forced expiratory volume in 3 and in 6 seconds $\left(\mathrm{FEV}_{3} / \mathrm{FEV}_{6}\right)(\mathrm{p}<0.05)$. In addition, the percentage of heavy smokers $(\geq 30$ pack/year) with R5 - R20 >0.07 $\mathrm{kPa} \cdot \mathrm{s} \cdot \mathrm{L}^{-1}$, considered as IOS index of SAD, but not with FEV3/FEV6 less than a lower limit of normal, a spirometry index of SAD, was significantly higher than that of mild smokers ( $<30$ pack/year) and never-smokers $(\mathrm{p}<0.05)$.

Conclusion: This study demonstrates that IOS has the potential to detect SAD in asymptomatic heavy smokers with preserved spirometry and with FEV3/FEV6 values in the normal range. We confirm that IOS provides parameters which can complement traditional measurements of pulmonary function.

Keywords: small airway dysfunction, impulse oscillometry system, spirometry, smokers, COPD

\section{Introduction}

Cigarette smoking is a well-known, causative factor for Chronic Obstructive Pulmonary Disease (COPD). Notably, it has been shown that smoking-induced bronchiolitis with progressive small airway dysfunction (SAD) is a leading cause of COPD. ${ }^{1}$

Since the lung has a wide functional reserve capacity, significant changes in pulmonary function may well occur before smokers complain of respiratory symptoms. It is possible for symptomatic smokers to exhibit preserved spirometry [Forced Expiratory Volume at 1st second $\left(\mathrm{FEV}_{1}\right)$ /Forced Vital Capacity (FVC) $\geq 0.70$ and FVC above the lower limit of the normal range], so that SAD can only be diagnosed by a high-resolution computed tomographic (HRCT) scan of the chest. $^{2}$ It is worth noting that FEV1 mainly reflects large airway obstruction,
Correspondence: Luigino Calzetta Department of Medicine and Surgery, Respiratory Disease and Lung Function Unit, University of Parma, Viale A. Gramsci, 14, Parma, 43126, Italy Email luigino.calzetta@unipr.it 
whereas later fraction of forced exhalation better reflects smaller airway patency and, thus, its measurement may be more sensitive in order to intercept reduction in terminal expiratory flows. ${ }^{3}$ Furthermore, the ratio of forced expiratory volume in 3 and in 6 seconds $\left(\mathrm{FEV}_{3} / \mathrm{FEV}_{6}\right)$ less than lower limit of normal (LLN) was the sole spirometric parameter able to identify a distinct population of current or ex-smokers with evidence of SAD at the quantitative CT scanning. ${ }^{4}$

Noteworthy is the fact that the Impulse Oscillometry System (IOS) measures the lungs' mechanical properties during quiet tidal breathing by the application of minimal pressures at the mouth. ${ }^{5}$ IOS may provide parameters which complement traditional measurements of pulmonary function, such as spirometry, lung volume, or diffusing capacity. ${ }^{5}$ The fall in resistance from $5 \mathrm{~Hz}$ to $20 \mathrm{~Hz}$ (R5 - R20) is considered a IOS measure of the small airways resistances. ${ }^{5,6}$ Intriguingly, IOS has the potential to detect SAD even in the setting of normal spirometry. ${ }^{6}$ Furthermore, in symptomatic smokers with spirometry in the normal range, peripheral airway dysfunction assessed by IOS parameters was significantly related to wheeze. ${ }^{7}$

The aim of this study was to investigate the value of IOS to detect SAD in a cohort of smokers showing preserved spirometry and not complaining of any chronic respiratory symptoms. We hypothesized that a smokinginduced SAD is already present even in these subjects and that it can be revealed by IOS. Furthermore, as a secondary outcome, we evaluated the relationship between R5 - R20 and FEV3/FEV6, both being simple and easy to perform tools for assessing small airway function.

\section{Methods}

\section{Study Design and Subjects}

This was a single-center, observational cross-sectional study conducted in a single session between October 2018 and December 2019 at the Lung Function Unit of University Hospital of Parma. We prospectively recruited individuals over 18 years of age, referred to the Center for smoking cessation of the University Hospital of Parma and who came to our Unit to receive pulmonary function testing.

We only included smokers with preserved spirometry and without respiratory symptoms or any history of pulmonary or cardiac disease, apart from well-controlled systemic hypertension. We also enrolled healthy, age and sexmatched subjects who had never smoked to serve as a control group.

Anthropometric variables, including age, sex, and body mass index (BMI) were collected in both groups of subjects as well as number of pack/year in the group of smokers. All subjects underwent IOS and spirometry, and smokers were instructed to abstain from smoking at least 1 $\mathrm{h}$ before testing. Written informed consent was obtained from all participants. The study protocol was approved by the regional ethics committee (Comitato Etico di Area Vasta Emilia Nord, Parma; approval number 36782; dated 28/09/2018).

\section{Oscillometry and Spirometry Measurements}

IOS was carried out according to current recommendations ${ }^{5}$ by means of the Jaeger MasterScreenIOS (CareFusion Technologies, San Diego, CA, USA), as previously described. ${ }^{8}$ Airway resistance detected at $5 \mathrm{~Hz}$ and $20 \mathrm{~Hz}$ (R5 and R20, expressed in $\mathrm{kPa} \cdot \mathrm{s} \cdot \mathrm{L}^{-1}$ ) represented indices of total and proximal airway resistance, respectively. The R5 - R20 value, ie, the index of the small airways resistances ${ }^{5,6}$ greater than $0.070 \mathrm{kPa} \cdot \mathrm{s} \cdot \mathrm{L}^{-1}$ was chosen to identify the presence of SAD. ${ }^{9-11}$ The reactance detected at $5 \mathrm{~Hz}\left(\mathrm{X} 5\right.$, expressed as $\left.\mathrm{kPa} \cdot \mathrm{s} \cdot \mathrm{L}^{-1}\right)$ and the integrated area of low-frequency reactance $(\mathrm{AX}$, expressed as $\mathrm{kPa} \cdot \mathrm{L}^{-1}$ ) from $5 \mathrm{~Hz}$ to resonant frequency (FRes, expressed as $\mathrm{Hz}$ ) were markers of peripheral airway abnormalities.

Spirometry was performed according to current international guidelines. ${ }^{12} \mathrm{FEV}_{1}, \mathrm{FVC}, \mathrm{FEV}_{1} / \mathrm{FVC}$ ratio, forced expiratory flow in the middle half of the exhaled volume (FEF25-75), and FEV3/FEV6 ratio were recorded.

Detailed information on methods concerning IOS and spirometry are reported in the Supplementary Data.

\section{Statistical Analysis}

The normal distribution of investigated variables was assessed via the Shapiro-Wilk test. Continuous variables characterized by non-normal distribution were reported as medians and 1 st and 3 rd quartiles. Continuous variables characterized by normal distribution were reported as means \pm standard deviation (SD). The number of patients and percentage were used as categorical variables.

The statistical analysis of continuous variables was performed via the test or the nonparametric Mann- 
Whitney test; the Bonferroni post-hoc analysis, the ANOVA, and the nonparametric Kruskal-Wallis test were carried out for multiple comparisons; the statistical analysis of categorical variables was carried out via the $\chi^{2}$ test, $\chi^{2}$ for trend test or Fisher exact test, as previously described. $^{13}$

Concerning the correlation analysis, the Pearson $(r)$ correlation coefficient was used for linear or normally distributed variables; the Spearman $(\rho)$ correlation coefficient was used for not linear or not normally distributed variables.

The statistical significance was set for $p$ value $<0.05$.

\section{Results}

We included 75 asymptomatic smokers (37 females, mean age $47 \pm 12$ years, $26 \pm 17$ pack/year) with preserved spirometry and 34 normal never-smokers (19 females, mean age $42 \pm 15$ years). Smokers had a significantly larger BMI $\left(25 \pm 3\right.$ vs $\left.24 \pm 3 \mathrm{Kg} / \mathrm{m}^{2}, p<0.05\right)$ and lower values of FVC ( $105 \pm 13$ vs $112 \pm 14 \%$ pred, $p<0.05)$, FEV1 (102 \pm 13 vs $107 \pm 13 \%, p<0.05)$ and FEV3/FEV6 $(0.94 \pm 0.02$ vs 0.96 $\pm 0.03, p<0.05)$, and higher values of R5-R20 (0.047 \pm 0.07 vs $\left.0.026 \pm 0.03 \mathrm{kPa} \cdot \mathrm{s} \cdot \mathrm{L}^{-1}, p<0.05\right)$ than normal neversmokers. In all smokers, pack/years were significantly related to spirometry (FVC, FEV1, FEV1/FVC, FEV3/ FEV6, FEF25-75) and IOS parameters (R5-R20, AX, FRes) (Table 1). In addition, in the same subjects, FEV3/ FEV6 values were significantly related to R5-R20, X5, AX and FRes values (Table 2).

When smokers were subdivided according to the number of pack/year in mild smokers $(<30)$ and in heavy smokers $(\geq 30)$, we found that heavy smokers were significantly older and had a higher BMI, as compared to mild

Table I Pearson $(r)$ or Spearman $(\rho)$ Correlation Coefficients and Corresponding $p$ values of Pack/Year When Related to Spirometry and IOS Parameters in 75 Smokers

\begin{tabular}{|l|c|c|}
\hline FVC (\% pred) & $r=-0.245$ & $\mathbf{p}=\mathbf{0 . 0 3 4}$ \\
FEVI (\% pred) & $r=-0.373$ & $\mathbf{p}=\mathbf{0 . 0 0 I}$ \\
FEVI/FVC (\%) & $r=-0.516$ & $\mathbf{p}=\mathbf{0 . 0 0 I}$ \\
FEF25-75 (\% pred) & $r=-0.414$ & $\mathbf{p}=\mathbf{0 . 0 0 I}$ \\
FEV3/FEV6 (\%) & $r=-0.516$ & $\mathbf{p}=\mathbf{0 . 0 0 I}$ \\
R5 $\left(\mathrm{kPa} \cdot \mathrm{s} \cdot \mathrm{L}^{-1}\right)$ & $\mathrm{r}=0.175$ & $\mathrm{P}=\mathrm{n} . \mathrm{s}$. \\
R20 $\left(\mathrm{kPa} \cdot \mathrm{s} \cdot \mathrm{L}^{-1}\right)$ & $\mathrm{r}=-0.049$ & $\mathrm{P}=\mathrm{n} . \mathrm{s}$. \\
R5-R20 $\left(\mathrm{kPa} \cdot \mathrm{s} \cdot \mathrm{L}^{-1}\right)$ & $\mathrm{r}=0.284$ & $\mathbf{p}=\mathbf{0 . 0 1 4}$ \\
AX $\left(\mathrm{kPa} \cdot \mathrm{L}^{-1}\right)$ & $\rho=0.235$ & $\mathbf{p}=\mathbf{0 . 0 4 2}$ \\
X5 $\left(\mathrm{kPa} \cdot \mathrm{s} \cdot \mathrm{L}^{-1}\right)$ & $\rho=-0.189$ & $\mathrm{p}=\mathrm{n} . \mathrm{s}$. \\
FRes $(\mathrm{Hz})$ & $\rho=0.283$ & $\mathbf{p}=\mathbf{0 . 0 1 4}$ \\
\hline
\end{tabular}

Note: Bold text indicates statistical significance.
Table 2 Pearson $(r)$ or Spearman $(\rho)$ Correlation Coefficients and Corresponding $p$ values of FEV3/FEV6 When Related to IOS Parameters in 75 Smokers

\begin{tabular}{|l|c|c|}
\hline R5 $\left(\mathrm{kPa} \cdot \mathrm{s} \cdot \mathrm{L}^{-1}\right)$ & $\mathrm{r}=-0.304$ & $\mathrm{P}=0.008$ \\
R20 $\left(\mathrm{kPa} \cdot \mathrm{s} \cdot \mathrm{L}^{-1}\right)$ & $\mathrm{r}=-0.181$ & $\mathrm{P}=\mathrm{n} . \mathrm{s}$. \\
R5-R20 $\left(\mathrm{kPa} \cdot \mathrm{s} \cdot \mathrm{L}^{-1}\right)$ & $\mathrm{r}=-0.333$ & $\mathbf{p}=\mathbf{0 . 0 0 3}$ \\
AX $\left(\mathrm{kPa} \cdot \mathrm{L}^{-1}\right)$ & $\rho=-0.293$ & $\mathbf{p}=\mathbf{0 . 0 1}$ \\
X5 $\left(\mathrm{kPa} \cdot \mathrm{s} \cdot \mathrm{L}^{-1}\right)$ & $\rho=0.252$ & $\mathrm{P}=0.029$ \\
FRes $(\mathrm{Hz})$ & $\rho=-0.310$ & $\mathbf{p}=\mathbf{0 . 0 0 7}$ \\
\hline
\end{tabular}

Note: Bold text indicates statistical significance.

smokers and healthy subjects (Table 3). Heavy smokers also differed significantly in spirometry and in IOS parameters when compared to mild smokers and healthy subjects (Table 3). Moreover, two subjects out of 34 healthy controls $(6 \%), 2$ out of 39 mild smokers $(5 \%)$, and 9 out of 36 heavy smokers (25\%) showed a R5 - R20 value $>0.07$ $\mathrm{kPa} \cdot \mathrm{s} \cdot \mathrm{L}^{-1}(p=0.013)$ (Figure 1$)$. In contrast, two subjects out of 34 healthy controls $(6 \%), 1$ out of 39 mild smokers $(2.5 \%)$, and 3 out of 36 heavy smokers (8\%) showed a FEV3/FEV6 value less than LNN ( $p=n s)$.

\section{Discussion}

The main finding of the present study is that IOS has the potential to detect SAD in asymptomatic heavy smokers with preserved spirometry. Notably, the percentage of heavy smokers $(\geq 30$ pack/year) with a R5 - R20 value $>0.07 \mathrm{kPa} \cdot \mathrm{s} \cdot \mathrm{L}^{-1}$, when considered an index of SAD, was significantly higher than that of normal never-smokers and mild smokers. In addition, R5-R20 values were significantly related to FEV3/FEV6, a spirometry parameter of SAD. However, the percentage of subjects with FEV3/ FEV6 values less than LLN in heavy smokers was not different than that of normal never-smokers. Lastly, we found that, in all smokers, the number of pack/year was significantly related both to IOS and spirometry parameters.

Previous studies showed that spirometry was less sensitive in detecting both small and large airway dysfunction, as compared to IOS across a broad spectrum of clinical and research settings. ${ }^{5,14-17}$ In a cohort of ironworkers exposed to the catastrophic events at the World Trade Center (WTC), Skloot et $\mathrm{al}^{14}$ showed that IOS revealed airway obstruction, expressed by abnormal R5 value, in $53 \%$ of subjects, while spirometry identified obstruction, defined by $\mathrm{FEV} 1 / \mathrm{FVC} \leq 0.70$ and $\mathrm{FEV} 1<80 \%$ pred, in only $17 \%$ of subjects. Tellingly, in symptomatic individuals with WTC dust/fume exposure and normal 
Table 3 Characteristics of Healthy Controls and Asymptomatic Mild (<30 Pack/Year) and Heavy ( $\geq 30$ Pack/Year) Smokers with Preserved Spirometry

\begin{tabular}{|c|c|c|c|c|}
\hline & Never-Smokers (No. 34) & Mild Smokers (No. 39) & Heavy Smokers (No. 36) & $\mathbf{p}$ \\
\hline Age (yr) & $42 \pm 15$ & $4 I \pm I I$ & $53 \pm 10$ & $<0.01 *$ \\
\hline Sex (F\%) & 56 & 46 & 53 & n.s. \\
\hline BMI $\left(\mathrm{Kg} / \mathrm{m}^{2}\right)$ & $24 \pm 3$ & $24 \pm 3$ & $27 \pm 4$ & $<0.01 *$ \\
\hline Pack/year & $\ldots$ & $14.5 \pm 7$ & $41.4 \pm 14$ & $\ldots$ \\
\hline FVC (\% pred) & $112 \pm 14$ & $107 \pm 12$ & $101 \pm 13$ & $<0.0 \mathrm{I}^{\#}$ \\
\hline FEVI (\% pred) & $107 \pm 13$ & $106 \pm 12$ & $97 \pm 13$ & $<0.01 *$ \\
\hline FEVI/FVC (\%) & $0.8 I \pm 0.07$ & $0.8 \mathrm{I} \pm 0.05$ & $0.76 \pm 0.05$ & $<0.01 *$ \\
\hline FEV3/FEV6 (\%) & $0.8 I \pm 0.07$ & $0.8 \mathrm{I} \pm 0.05$ & $0.76 \pm 0.05$ & $<0.01 *$ \\
\hline FEF25-75 (\% pred) & $83 \pm 25$ & $95 \pm 24$ & $72 \pm 30$ & $<0.01^{\S}$ \\
\hline $\mathrm{R} 5\left(\mathrm{kPa} \cdot \mathrm{s} \cdot \mathrm{L}^{-1}\right)$ & $0.348 \pm 0.09$ & $0.35 I \pm 0.09$ & $0.426 \pm 0.16$ & $<0.05 *$ \\
\hline $\mathrm{R} 20\left(\mathrm{kPa} \cdot \mathrm{s} \cdot \mathrm{L}^{-1}\right)$ & $0.322 \pm 0.07$ & $0.326 \pm 0.08$ & $0.354 \pm 0.10$ & n.s. \\
\hline R5-R20 (kPa s $\left.\cdot \mathrm{L}^{-1}\right)$ & $0.026 \pm 0.03$ & $0.025 \pm 0.03$ & $0.07 I \pm 0.09$ & $<0.01 *$ \\
\hline $\mathrm{AX}\left(\mathrm{kPa} \cdot \mathrm{L}^{-1}\right)$ & $0.20[0.14-0.34]$ & $0.20[0.12-0.37]$ & $0.32[0.16-0.81]$ & n.s. \\
\hline $\mathrm{X} 5\left(\mathrm{kPa} \cdot \mathrm{s} \cdot \mathrm{L}^{-1}\right)$ & $-0.09[-0.11-0.07]$ & $-0.09[-0.12-0.07]$ & $-0.11[-0.15-0.08]$ & $<0.05 *$ \\
\hline FRes $(\mathrm{Hz})$ & $10.6[9.0-13.8]$ & 10.3 [8.9-14.8] & 14.9 [9.2-18.4] & n.s. \\
\hline
\end{tabular}

Notes: Values are expressed as mean \pm SD, median [25-75\% percentile] or \%. Bold text indicates statistical significance. *Heavy Smokers vs Healthy Subjects and Mild Smokers, \#Heavy Smokers vs Healthy Subjects, §Heavy Smokers vs Mild Smokers.

spirometry, Oppenheimer et $\mathrm{al}^{9}$ found both elevated global airway resistance and heterogeneity of distal airway function, demonstrated by IOS. In addition, in subjects with chronic respiratory symptoms and normal spirometry, IOS was more sensitive in revealing SAD and correlated better with symptoms than spirometry. ${ }^{15}$ In patients with COPD, IOS parameters showed a better correlation with small airway changes assessed by endo-bronchial optical coherence tomography than spirometry. ${ }^{16}$ In asthmatic patients with normal FEV1 values, IOS revealed SAD, which was found to be associated with poor disease control and a history of asthma exacerbations. ${ }^{17}$ The results of the present study, in showing a SAD revealed by IOS in asymptomatic smokers with preserved spirometry, further confirm and extend the previous findings.

In all smokers, we found a significant inverse correlation between pack/years and spirometry and a direct significant correlation between pack/years and IOS parameters. Though correlation does not imply causality, we can speculate that a smoking habit has already had deleterious effects on the pulmonary function of smokers without respiratory symptoms and with preserved spirometry. It is of note that in mild smokers with as little as 10 pack/years smoking history, an impairment in both the conductive and acinar lung zone compartments of the lung, assessed by multiple breath washout test, was found. ${ }^{18}$ Furthermore, some histologic aspects of respiratory bronchiolitis were found in young smokers who died a sudden non-hospital death. ${ }^{19}$
In the present study, we found that heavy smokers were older, with higher BMI, lower values of spirometry, and higher values of total and peripheral resistances by IOS than mild smokers, but they were not different in sex. We cannot exclude that being overweight and mild obesity, which were more prevalent in the heavy smokers, significantly influenced per se pulmonary function in these patients. It is worth noting that an increased cigarette consumption among smokers was found to be significantly associated with higher $\mathrm{BMI}^{20}$ and that physical inactivity might be a poor behavioral habit mediating the smokingBMI connection. ${ }^{20,21}$ Notably, in this study we found a direct significant correlation between pack/years and BMI (data not shown).

Among spirometry parameters, a reduced FEF25-75 is considered to be an index of airway obstruction in peripheral bronchioles. ${ }^{22}$ Accordingly, small airway dysfunction is commonly diagnosed on the basis of FEF25-75 value less than $60 \%{ }^{10}$ or $65 \%^{23}$ of predicted value, in relation to different clinical settings. However, the wide range of normal values of FEF25-75, as compared to other spirometry index, causes an unacceptably great number of false-positives and false negatives in the assessment of SAD. ${ }^{3}$ By contrast, the spirometry parameters for assessing the later fraction of forced exhalation, such as FEV3/ FEV6, are considered reliable measurements of peripheral airway patency. ${ }^{3,4}$ In the present study, we found that FEV3/FEV6 was strictly related to all IOS markers of 

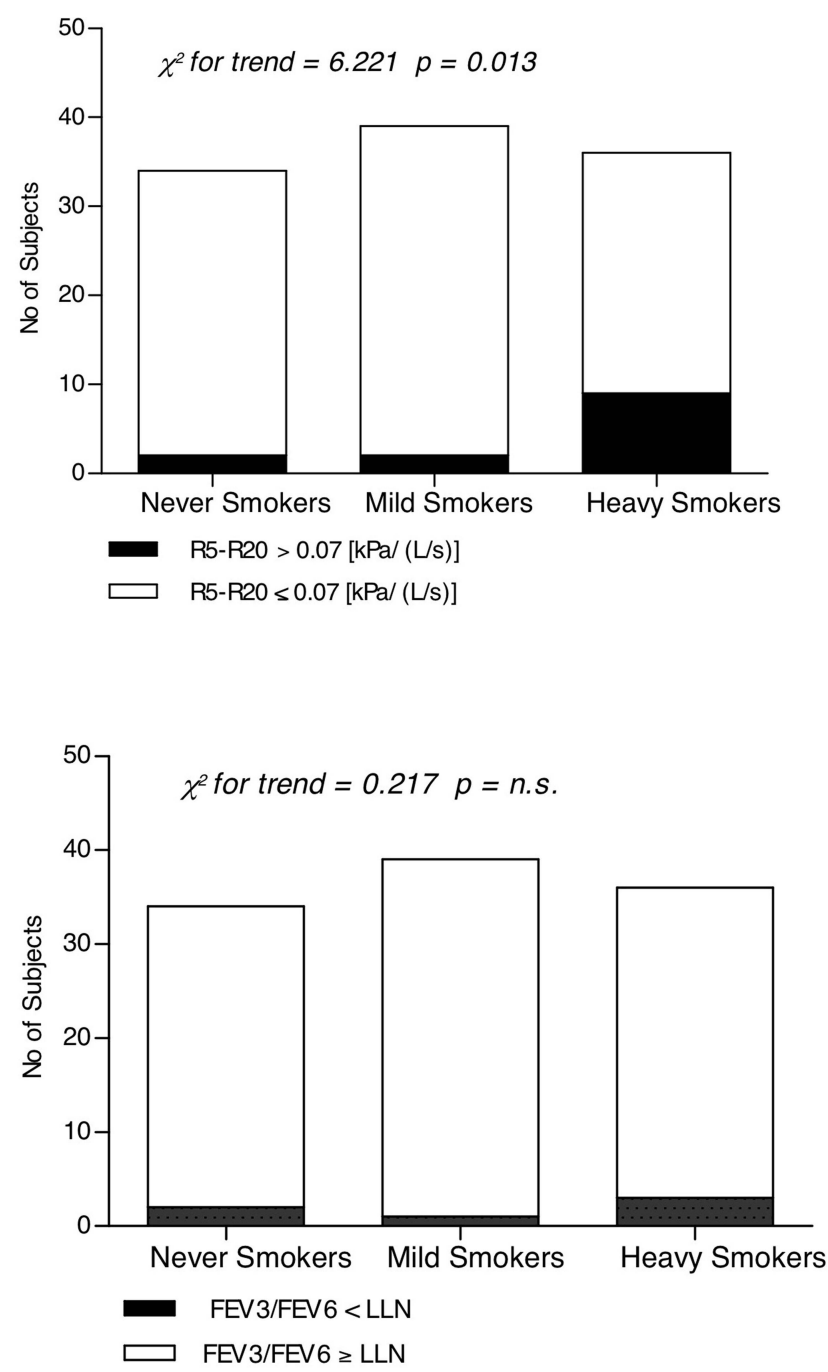

Figure I Number of subjects categorized according to the presence of SAD (R5 $\mathrm{R} 20>0.07 \mathrm{kPa} \cdot \mathrm{s}^{-1}$ ) in 34 normal never-smokers, in 39 mild smokers (pack/year < 30 ), and in 36 heavy smokers (pack/year $\geq 30$ ).

peripheral airway abnormalities. However, unlike R5-R20, FEV3/FEV6 was not able to intercept SAD among asymptomatic smokers with spirometry in the normal range. The greater diagnostic sensitivity of IOS compared to spirometry could be due to the greater simplicity in performing IOS, which requires the subject to simply breathe at tidal volume without any specific cooperation.

Some limitations should be acknowledged in this study. First, IOS measurement is still lacking universally accepted normal references values; therefore, we used only the absolute values and not those expressed as the percentage of the predicted value when we compared smokers and normal control subjects. Studies on large samples of normal healthy populations are needed to obtain IOS reference values. Secondly, due to cross-sectional nature of this study, we cannot predict that smokers with SAD will become COPD patients. Further long-term follow-up studies are required to investigate the relationship between SAD, assessed by IOS, and the development of COPD. Lastly, in this study we used IOS and spirometry to early identify SAD in smokers. CT scans $^{2}$ and nitrogen washouts ${ }^{18}$ have been advocated for early detection of peripheral airways disease in smokers with preserved spirometry; however, they are not relevant for routinely detecting early signs of SAD and mainly remain research tools without practical, economical, or a safety rationale at this stage.

\section{Conclusion}

We conclude that asymptomatic smokers with spirometry in the normal range may have SAD, which can be detected by IOS. The present study further confirms that IOS parameters can complement traditional measurements of pulmonary function and suggest that IOS is a promising tool for the early diagnosis of COPD in subjects at risk.

\section{Abbreviations}

AX, area of low-frequency reactance; BMI, body mass index; FEF25-75, forced expiratory flow in the middle half of the patient's exhaled volume; $\mathrm{FEV}_{1}$, Forced Expiratory Volume at 1st second; Fres, resonant frequency; FVC, Forced Vital Capacity; HRCT, highresolution computed tomography; IOS, the Impulse Oscillometry System; R5 - R20, fall in resistance from 5 $\mathrm{Hz}$ to $20 \mathrm{~Hz}$; SAD, small airway dysfunction.

\section{Data Sharing Statement}

Not applicable, there are no unpublished data from the study.

\section{Ethics Approval and Inform Consent}

The study protocol was approved by the local ethical committee (approval number 36782; dated 28/09/2018). Written informed consent was obtained from all participants. This study was conducted in accordance with the Declaration of Helsinki.

\section{Author Contributions}

All authors made a significant contribution to the work reported, whether that was in the conception, study design, execution, acquisition of data, analysis and interpretation, or in all these areas; took part in drafting, revising or critically reviewing the article; gave final approval of the version to be published; have agreed on the journal to 
which the article has been submitted; and agree to be accountable for all aspects of the work. All authors have read and approved the final manuscript.

\section{Funding}

This study was not funded.

\section{Disclosure}

The authors declare that they have no conflicts of interest for this work.

\section{References}

1. McDonough JE, Yuan R, Suzuki M, et al. Small-airway obstruction and emphysema in chronic obstructive pulmonary disease. $N$ Engl $J$ Med. 2011;365(17):1567-1575. doi:10.1056/nejmoa1106955

2. Woodruff PG, Barr RG, Bleecker E, et al. Clinical significance of symptoms in smokers with preserved pulmonary function. $N$ Engl J Med. 2016;374(19):1811-1821. doi:10.1056/nejmoa1505971

3. Hansen JE, Sun XG, Wasserman K. Discriminating measures and normal values for expiratory obstruction. Chest. 2006;129 (2):369-377. doi:10.1378/chest.129.2.369

4. Dilektasli AG, Porszasz J, Casaburi R, et al.; COPDGene investigators. A novel spirometric measure identifies mild COPD unidentified by standard criteria. Chest. 2016;150(5):1080-1090. doi:10.1016/j. chest.2016.06.047

5. Thamrin C, Dellacà RL, Hall GL, et al. Technical standards for respiratory oscillometry: test loads for calibration and verification. Eur Respir J. 2020;56(4):2003369. doi:10.1183/13993003.033692020

6. Bickel S, Popler J, Lesnick B, Eid N. Impulse oscillometry: interpretation and practical applications. Chest. 2014;146(3):841-847. doi:10.1378/chest.13-1875

7. Jetmalani K, Thamrin C, Farah CS, et al. Peripheral airway dysfunction and relationship with symptoms in smokers with preserved spirometry. Respirology. 2018;23(5):512-518. doi:10.1111/ resp. 13215

8. Pisi R, Aiello M, Zanini A, et al. Small airway dysfunction and flow and volume bronchodilator responsiveness in patients with chronic obstructive pulmonary disease. Int J COPD. 2015;10:1191-1197. doi:10.2147/COPD.S82509

9. Oppenheimer BW, Goldring RM, Herberg ME, et al. Distal airway function in symptomatic subjects with normal spirometry following world trade center dust exposure. Chest. 2007;132(4):1275-1282. doi:10.1378/chest.07-0913

10. Lipworth B, Manoharan A, Anderson W. Unlocking the quiet zone: the small airway asthma phenotype. Lancet Respir Med. 2014;2 (6):497-506. doi:10.1016/s2213-2600(14)70103-1
11. Crisafulli E, Pisi R, Aiello M, et al. Prevalence of small-airway dysfunction among COPD patients with different GOLD stages and its role in the impact of disease. Respiration. 2016;93(1):32-41. doi: $10.1159 / 000452479$

12. Graham BL, Steenbruggen I, Barjaktarevic IZ, et al. Standardization of spirometry 2019 update an official American Thoracic Society and European Respiratory Society technical statement. Am J Respir Crit Care Med. 2019;200(8):E70-E88. doi:10.1164/rccm.201908-1590ST

13. Crisafulli E, Teopompi E, Luceri $S$, et al. The value of high-resolution computed tomography (HRCT) to determine exercise ventilatory inefficiency and dynamic hyperinflation in adult patients with cystic fibrosis. Respir Res. 2019;20(1):1. doi:10.1186/s12931019-1044-8

14. Skloot G, Goldman M, Fischler D, et al. Respiratory symptoms and physiologic assessment of ironworkers at the World Trade Center disaster site. Chest. 2004;125(4):1248-1255. doi:10.1378/ chest.125.4.1248

15. Li LY, Yan TS, Yang J, et al. Impulse oscillometry for detection of small airway dysfunction in subjects with chronic respiratory symptoms and preserved pulmonary function. Respir Res. 2021;22 (1):1-10. doi:10.1186/s12931-021-01662-7

16. Su ZQ, Guan WJ, Li SY, et al. Significances of spirometry and impulse oscillometry for detecting small airway disorders assessed with endobronchial optical coherence tomography in COPD. Int J COPD. 2018;13:3031-3044. doi:10.2147/COPD.S172639

17. Pisi R, Tzani P, Aiello M, et al. Small airway dysfunction by impulse oscillometry in asthmatic patients with normal forced expiratory volume in the 1st second values. Allergy Asthma Proc. 2013;34 (1):14-20. doi:10.2500/aap.2013.34.3641

18. Verbanck S, Schuermans D, Meysman M, Paiva M, Vincken W. Noninvasive assessment of airway alterations in smokers: the small airways revisited. Am J Respir Crit Care Med. 2004;170(4):414-419. doi:10.1164/rccm.200401-037OC

19. Niewoehner DE, Kleinerman J, Rice DB. Pathologic changes in the peripheral airways of young cigarette smokers. $N$ Engl $J$ Med. 1974;291(15):755-758. doi:10.1056/nejm197410102911503

20. Dvorak RD, Del Gaizo AL, Engdahl RM, Eliason CJ. Tobacco use and body mass index: mediated effects through physical inactivity. $J$ Health Psychol. 2009;14(7):919-923. doi:10.1177/ 1359105309341005

21. Panagiotakos DB, Pitsavos C, Lentzas Y, et al. Determinants of physical inactivity among men and women from Greece: a 5-year follow-up of the ATTICA study. Ann Epidemiol. 2008;18 (5):387-394. doi:10.1016/j.annepidem.2007.11.002

22. McFadden ER, Linden DA. A reduction in maximum mid-expiratory flow rate. A spirographic manifestation of small airway disease. Am J Med. 1972;52(6):725-737. doi:10.1016/0002-9343(72)90078-2

23. Xiao D, Chen Z, Wu S, et al. Prevalence and risk factors of small airway dysfunction, and association with smoking, in China: findings from a national cross-sectional study. Lancet Respir Med. 2020;8 (11):1081-1093. doi:10.1016/S2213-2600(20)30155-7

\section{Publish your work in this journal}

The International Journal of COPD is an international, peer-reviewed journal of therapeutics and pharmacology focusing on concise rapid reporting of clinical studies and reviews in COPD. Special focus is given to the pathophysiological processes underlying the disease, intervention programs, patient focused education, and self management protocols. This journal is indexed on PubMed Central, MedLine and CAS. The manuscript management system is completely online and includes a very quick and fair peer-review system, which is all easy to use. Visit http://www.dovepress.com/testimonials.php to read real quotes from published authors. 